\title{
Acute exacerbation of idiopathic pulmonary fibrosis
}

\author{
Lee Fidler MD, Shane Shapera MD
}

A 70-year-old-woman with idiopathic pulmonary fibrosis (IPF) presented with worsening dyspnea, nonproductive cough and increasing home oxygen requirements over a $24 \mathrm{~h}$ period.

IPF had been previously diagnosed in accordance with American Thoracic Society guidelines (1); having a consistent clinical presentation, absence of alternative diagnosis and a definite usual interstitial pneumonia pattern on chest computed tomography (CT). History included hemochromatosis, diabetes, hypertension and osteoarthritis. Pirfenidone was initiated two months before presentation. Physical examination revealed clubbing and bilateral basal-predominant fine crackles, consistent with IPF without evidence of heart failure.

A routine CT scan of the chest had been performed two days before developing increased dyspnea (Figure 1A) and a repeat contrastenhanced CT chest scan at the time of hospitalization (Figure 1B). There was interval development of diffuse ground-glass opacities (GGO) in both lungs without evidence of pulmonary embolism. Sputum cultures and a nasopharyngeal swab (for polymerase chain reaction) failed to identify bacterial, fungal or viral infection. Bronchoscopy could not be performed due to high oxygen requirements.

Treatment with $1 \mathrm{~g}$ of intravenous methylprednisilone was given for three days, followed by $50 \mathrm{mg}$ of prednisone. Dyspnea and oxygen requirements returned to baseline. Repeat imaging at one week (Figure 1C) showed interval resolution of GGO. The patient was discharged home with plans to taper off her prednisone over a four-week period.

\section{KEY LEARNING POINTS}

- Acute exacerbations of IPF (AE-IPF) are characterized by (2):

- Previous or concurrent diagnosis of IPF;

- Unexplained worsening dyspnea within 30 days;

- CT showing new GGO and/or consolidation on a background usual interstitial pneumonia pattern;

- Absence of pulmonary infection on endotracheal aspirate or bronchoalveolar lavage; and

- Absence of an alternative cause including left heart failure or pulmonary embolism.

- AE-IPF is associated with a high mortality (2).

- Treatment for AE-IPF with high-dose corticosteroids is recommended with very low-quality evidence (1) and can lead to clinical and radiographic improvement.

\section{REFERENCES}

1. Raghu G, Collard HR, Egan JJ, et al. An official ATS/ERS/JRS/ ALAT statement: Idiopathic pulmonary fibrosis: Evidence-based guidelines for diagnosis and management. Am J Respir Crit Care Med 2011;183:788-824.

2. Collard HR, Moore BB, Flaherty KR, et al. Acute exacerbations of idiopathic pulmonary fibrosis. Am J Respir Crit Care Med 2007;176:636-43.

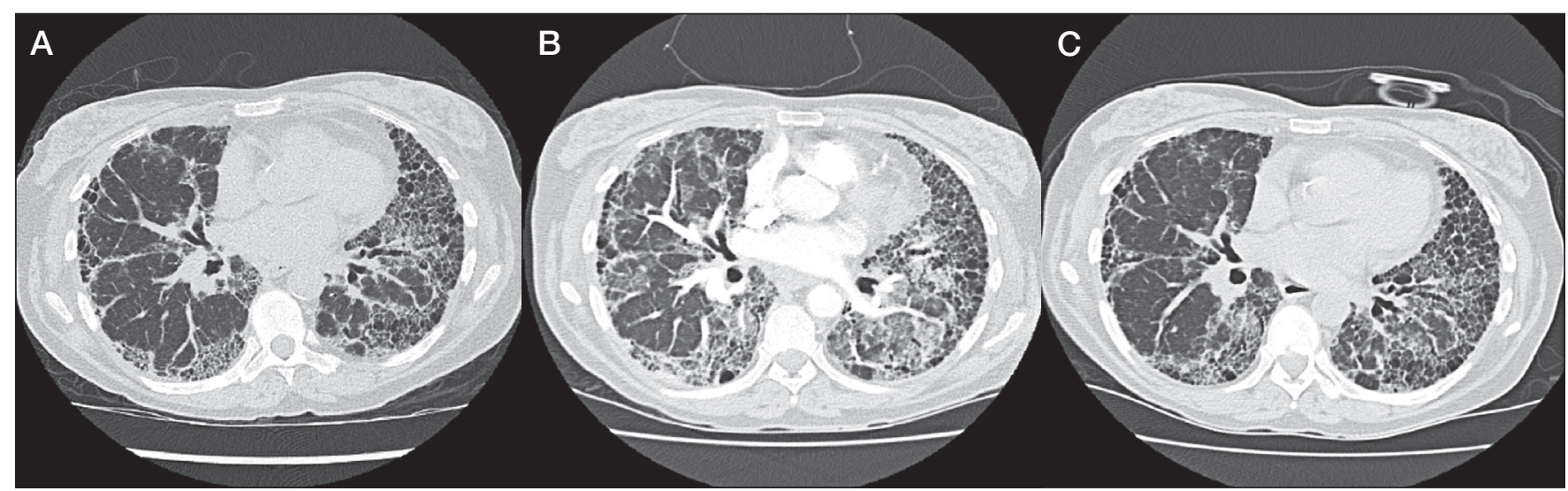

Figure 1) Computed tomography (CT) of the chest at baseline (A) revealing subpleural reticulation of basilar predominance and honeycombing, consistent with a definite usual interstitial pneumonia pattern. Repeat CT imaging, with increased ground-glass opacities, performed at initial presentation of idiopathic pulmonary fibrosis exacerbation (B) and improvement following one week of steroid therapy (C)

University of Toronto, Department of Medicine, Division of Respirology, Toronto, Ontario

Correspondance: Dr Lee Fidler, University of Toronto, Department of Medicine, Division of Respirology, 1 King's College Circle,

6263 Medical Sciences Building, Toronto, Ontario M5S 1A8. Telephone 416-999-1384, e-mail lee.fidler@utoronto.ca 


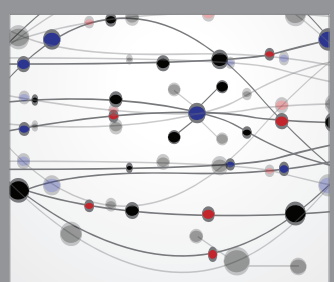

The Scientific World Journal
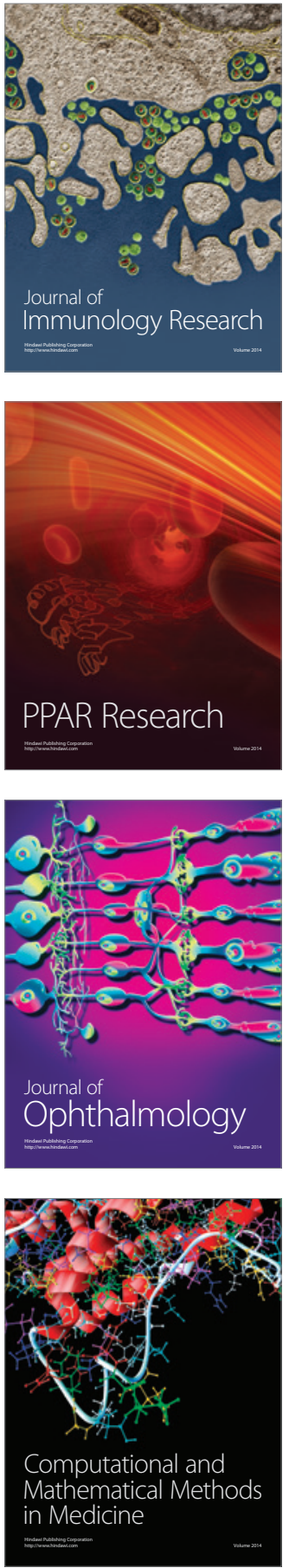

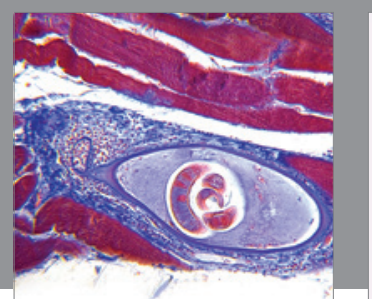

Gastroenterology Research and Practice

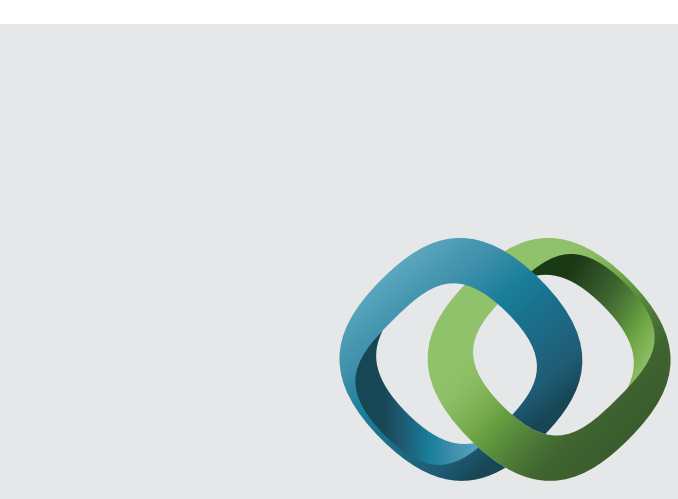

\section{Hindawi}

Submit your manuscripts at

http://www.hindawi.com
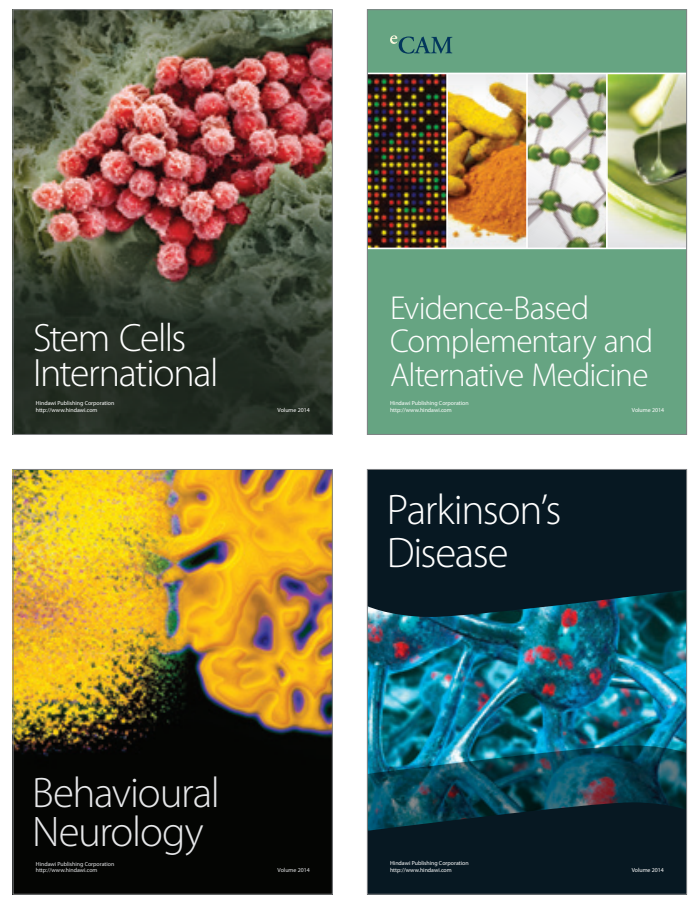
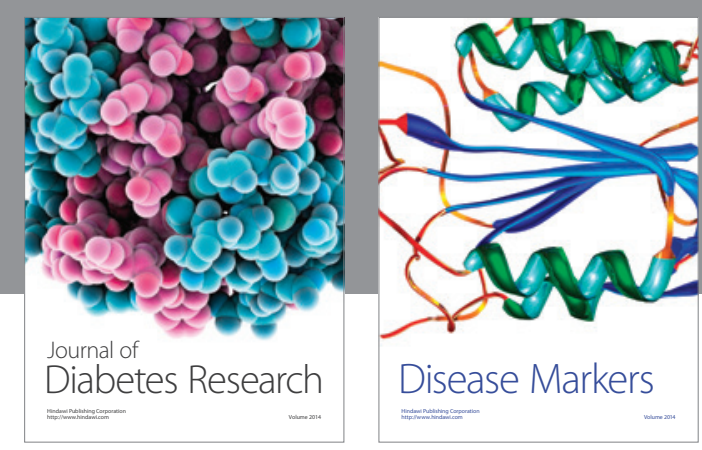

Disease Markers
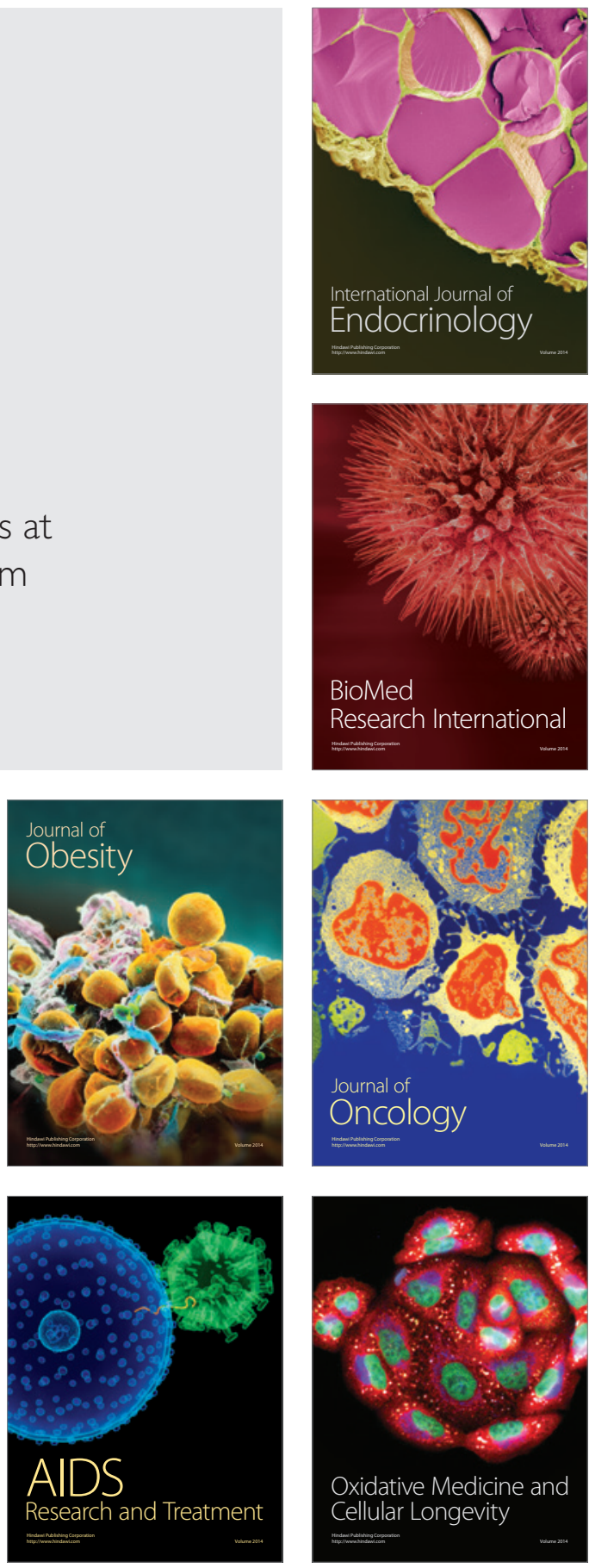